Conclusion Simple clinical criteria can be used to identify low-risk patients suitable for very early discharge $48 \mathrm{~h}$ following uncomplicated successful primary PPCI. With only a small percentage of complications occurring after the first $24 \mathrm{~h}$, discharge after $24 \mathrm{~h}$ may be safe and warrants further study.

\section{THE ROLE OF THE NEURAL NETWORKS IN IDENTIFICATION AND ABLATION OF PULMONARY VEIN ECTOPIC TRIGGERS}

\author{
doi:10.1136/heartjnl-2012-301877b.49
}

L Malcolme-Lawes, ${ }^{*}$ P B Lim, I Wright, P Kojodjojo, M Koa-Wing, S Jamil-Copley, N S Peters, D W Davies, P Kanagaratnam. Imperial College London, London, UK

Introduction The cardiac neural network comprises ganglionated plexi (GP) adjacent to each pulmonary vein (PV) which can trigger $\mathrm{AV}$ block and PV ectopy. We hypothesised that AV block in response to GP stimulation is mediated through the right lower GP (RLGP) and could be abolished by ablation of the RLGP, whereas a local PV ectopy response could be abolished by selective local GP ablation.

Methods and Results 10 patients undergoing left atrial (LA) ablation with autonomic modification were recruited. Continuous HFS was delivered endocardially at presumed GP sites using a Grass Stimulator $(20 \mathrm{~Hz}, 10 \mathrm{~ms}$ pulse duration, $10 \mathrm{~V})$. GP sites with connections to the AV node (producing AV block) were marked on LA geometry. RLGP group ( $N=5)$ had RF ablation of RLGP and 2 of 15 sites remained positive $(p<0.001)$ demonstrating that ablation of the RLGP can disrupt the neural connections to the AV node. The other group ( $\mathrm{N}=5$ ) had RF ablation of non-RLGP sites followed by HFS retesting of all previously positive unablated sites. All 19 unablated GP sites were positive after ablation of target the GP site indicating that the neural connections to the AV node remained intact. A further 18 patients in sinus rhythm at the time of AF ablation were recruited. Short bursts of HFS $(12 \mathrm{~V}, 50 \mathrm{~Hz}, 10 \mathrm{~ms}$ pulse width), synchronised to local refractory period, were delivered at presumed GP sites during fixed rate pacing. Positive sites initiating PV ectopy were recorded on the LA geometry and presumed to have PV connections. In a control group $(\mathrm{N}=3)$ to check reproducibility, 10 positive sites were identified and all 10 remained positive following catheter relocation and retesting. In the PVI group $(\mathrm{N}=8)$, 36 sites were positive, PVI was performed in all four veins without targeting GP sites during ablation. No sites were positive on retesting proving neural connections to the PV could be disrupted by ablation between the GP and PV. Finally in the GP ablation group $(\mathrm{N}=7), 29$ sites were positive, two patients remained in AF, therefore further testing was not possible. RF ablation was performed around positive site and on retesting, five of 27 sites were positive. After additional RF ablation at these sites, no further ectopy could be initiated on retesting. This indicated that the GP connections into PVs could also be rendered ineffective by GP ablation.
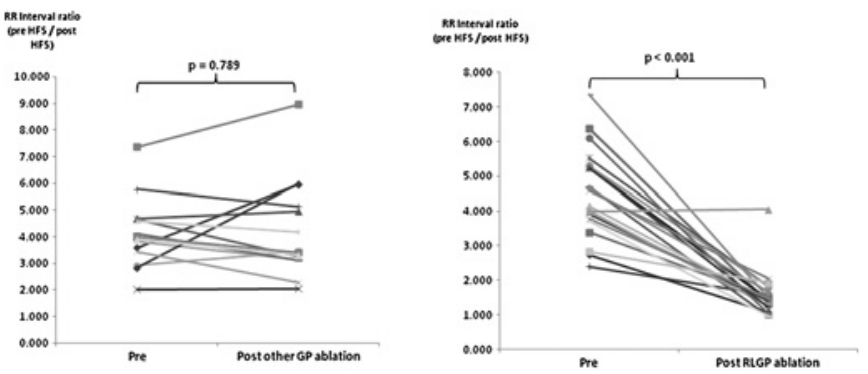

Abstract 049 Figure 1 Effect of other GP site ablation on RR interval change following HFS at distant GP sites. Effect of RLGP ablation on RR interval change following HFS at distant GP sites.
Conclusions Our study shows that neural inputs from LA GPs act on the AV node via the RLGP "gateway" and if AV block is used to identify GP sites for autonomic modulation, intact connections via the RLGP are required. We propose using synchronised HFS to identify and ablate GP sites initiating ectopic firing from the PV, as it only requires intact local connections to the PV. PVI acutely prevents this ectopic activity, possibly by transecting neural inputs to the veins. However, reconnection of PVs is common and RF ablation of the GP site alone may prevent ectopic firing within PVs.

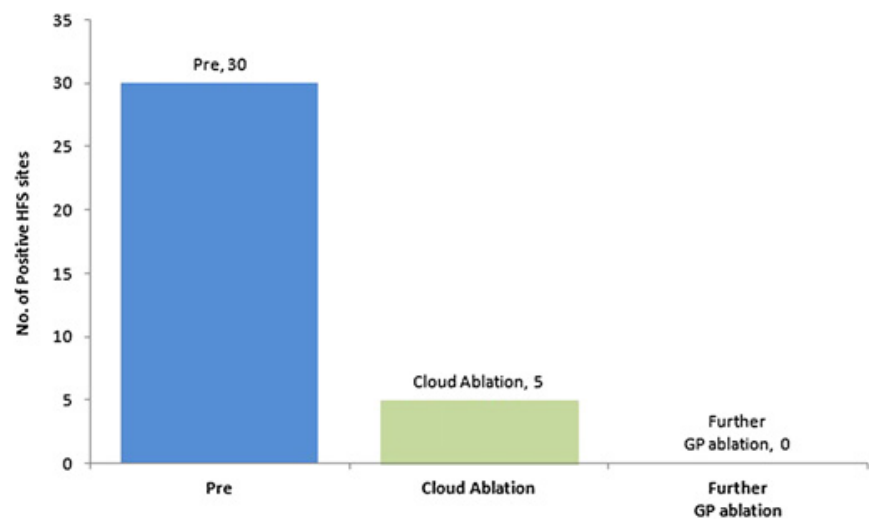

Abstract 049 Figure 2 Number of sites initiating PV ectopy before and after intervention in the GP ablation group.

\section{AUTOMATED ANALYSIS OF ATRIAL DELAYED ENHANCEMENT CARDIAC MRI CORRELATES WITH VOLTAGE, AF RECURRENCE POST-ABLATION, AND HIGH STROKE RISK}

doi:10.1136/heartjnl-2012-301877b.50

${ }^{1} \mathrm{~L}$ Malcolme-Lawes, ${ }^{* 1} \mathrm{C}$ Juli, ${ }^{1} \mathrm{R}$ Karim, ${ }^{1} \mathrm{~W}$ Bai, ${ }^{1} \mathrm{R}$ Quest, ${ }^{1} \mathrm{P}$ B Lim, ${ }^{1} \mathrm{~S}$ Jamil-Copley, ${ }^{1} \mathrm{P}$ Kojodjojo, ${ }^{1} \mathrm{~B}$ Ariff, ${ }^{1} \mathrm{D} W$ Davies, ${ }^{1} \mathrm{D}$ Rueckert, ${ }^{2} \mathrm{R}$ Hunter, ${ }^{2} \mathrm{R}$ Boubertakh, ${ }^{2} \mathrm{~S}$ Petersen, ${ }^{2} \mathrm{R}$ Schilling, ${ }^{1} \mathrm{P}$ Kanagaratnam, ${ }^{1} \mathrm{~N}$ S Peters. ${ }^{1}$ Imperial College London, London, UK; ${ }^{2}$ Barts and the London Biomedical Research Unit, London, UK

Introduction Visualisation of atrial scar using delayed-enhanced MRI (DE-MRI) may reveal causes for atrial fibrillation recurrence following ablation. To develop an objective method for delineating

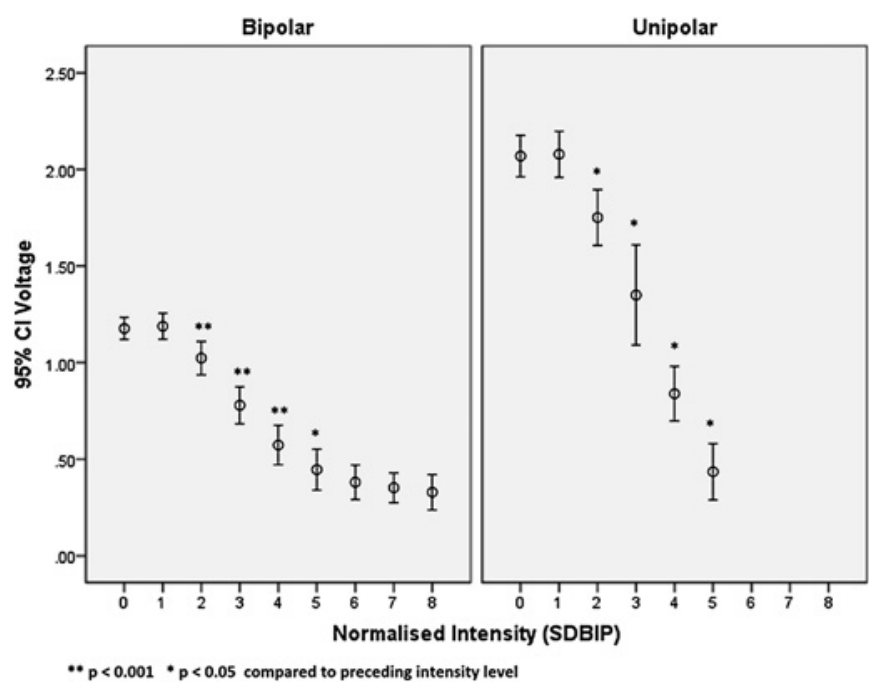

Abstract 050 Figure 1 Voltage type. ${ }^{*} p<0.001,{ }^{*} p<0.005$ compared to preceding intensity level. 


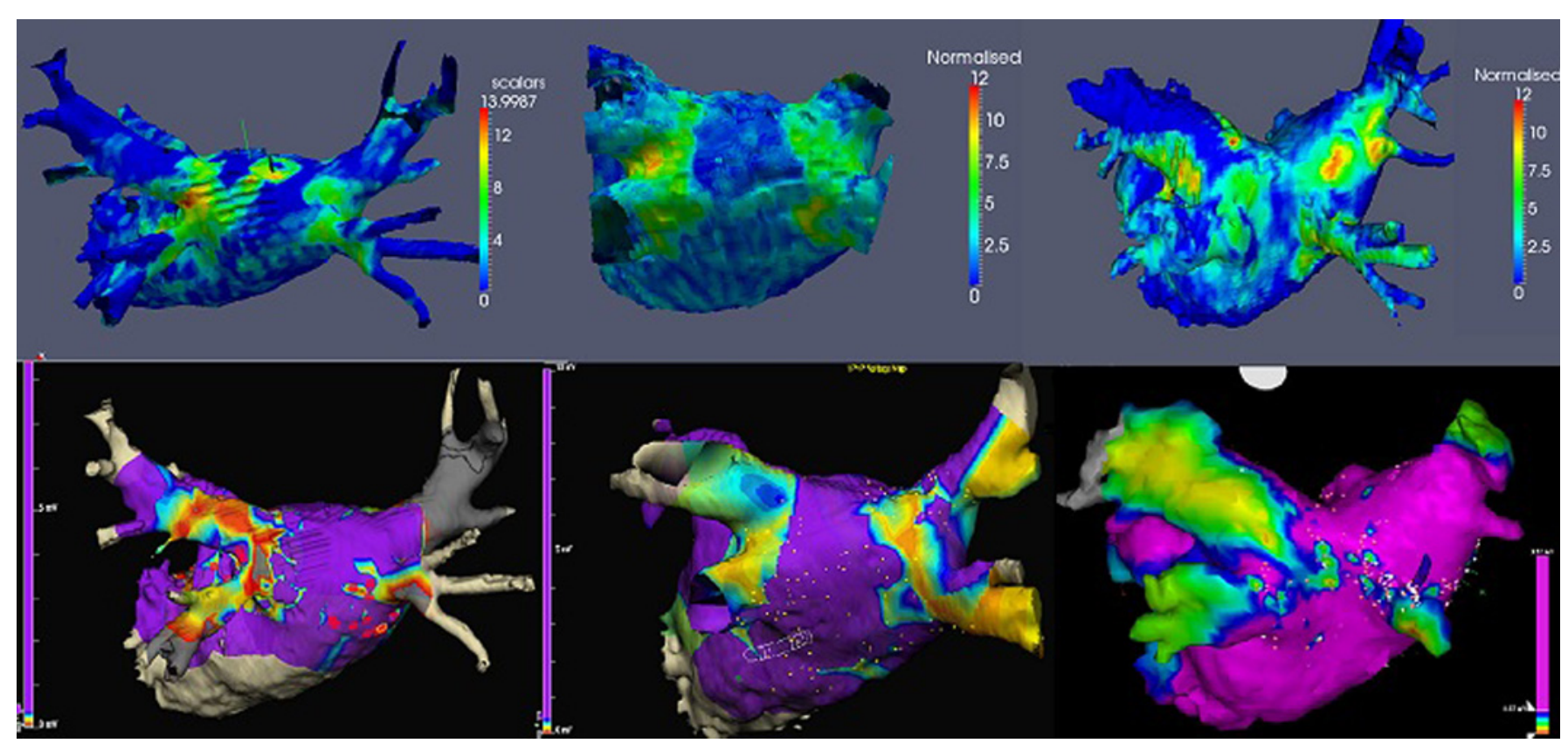

Abstract 050 Figure 2

ablation-scar we compared DE-MRI before and after Cryoballoon ablation on the basis that a predictable antral lesion set would be created. The method was subsequently used in patients undergoing radiofrequency or cryoballoon ablation. Endocardial voltage was measured and compared with corresponding enhancement levels on DE-MRI. Pre and post ablation enhancement was then compared with clinical outcome and risk factors for stroke.

Methods Pre-ablation DE-MRI was performed in 50 patients from two centres, undergoing pulmonary vein isolation; 25 with Arctic Front cryoballoon and 25 with conventional circumferential pulmonary vein ablation. Post-ablation DE-MRI was performed at 3 months. 10 patients at the first procedure and 12 patients returning for a redo procedure underwent endocardial voltage mapping with registration to MRA segmentation for comparison with the DE-MRI. The free-breathing late gadolinium enhanced sequence was also registered to the MRA segmentation and surface intensities were normalised using mean intensity of the blood pool (BIP). Normalised intensity levels were projected onto LA surface as multiples of SD above BIP mean.

Results Ostial and LA intensity were similar in pre ablation scans and greater in the ostia in post ablation scans ( $p=0.48$ and $p<0.001$ respectively). Bipolar voltage measurements were compared for each intensity level. No significant differences were noted between SD 0 and 1, however significant difference were noted between SD 1 and $2(1.41 \pm 0.03$ vs $1.26 \pm 0.04 \mathrm{mV} \mathrm{p}<0.001)$, SD 2 and $3,(1.26 \pm 0.04$ vs $0.90 \pm 0.05 \mathrm{mV} \mathrm{p}<0.001), \mathrm{SD} 3$ and $4(0.90 \pm 0.05$ vs $0.63 \pm 0.04 \mathrm{mV}$ $\mathrm{p}<0.001)$ and SD 4 and $5,(0.63 \pm 0.04$ vs $0.45 \pm 0.05 p=0.004)$. No significant differences were found between intensities $>$ SD 5 . Intensity $>$ SD 3 was identified as low voltage atrial tissue $<1 \mathrm{mV}$ (scar). \% scar was higher in patients with high risk of stroke $\left(\mathrm{CHADS}_{2}>1\right)$ compared to low $\left(\mathrm{CHADS}_{2}=0\right)$, (low vs high $3.19 \pm 3.17 \%$ vs $7.13 \pm 7.38 \% p=0.035)$. \% scar was also greater in pre ablation scans from patients with AF recurrence vs none following ablation $(6.6 \pm 6.7 \%$ vs $3.5 \pm 3.0 \% \mathrm{p}=0.032)$.

Conclusion We have demonstrated the feasibility of an objective, automated method of DE-MRI analysis of left atrial ablation-scar. Atrial enhancement is reflective of endocardial low-voltage myocardium over a range of intensities and voltages. Pre-ablation atrial scar identified patients at higher risk of stroke and reduced success from AF ablation, suggesting potential future roles for DE-MRI in the management of patients with AF.

\section{ORGANISATIONAL INDEX IS THE BEST ASSESSMENT OF FRACTIONATION IN PERSISTENT ATRIAL FIBRILLATION: ANALYTIC COMPARISONS AND ABLATION RESULTS}

doi:10.1136/heartjnl-2012-301877b.51

${ }^{1} \mathrm{M}$ C Finlay, ${ }^{*} \mathrm{~B}$ Lim, ${ }^{2} \mathrm{~J}$ McCready, ${ }^{2} \mathrm{~S}$ Ahsan, ${ }^{2} \mathrm{~A}$ B Gopalmurugan, ${ }^{3} \mathrm{~L} \mathrm{Xu},{ }^{3} \mathrm{O}$ Segal, ${ }^{2} \mathrm{M}$ Lowe, ${ }^{2} \mathrm{P} D$ Lambiase. ${ }^{1} U C L$, The Heart Hospital, UK; ${ }^{2} U C L H, U K ;{ }^{3} U C L$, UK

Introduction Targeting fractionated electrograms (EGMs) is commonly used in catheter ablation (CA) of persistent atrial fibrillation (PersAF). Several automated algorithms exist to identify sites of fractionation. EGMs exhibiting continuous activity (ContA) and a high organisation index (OI) have also been proposed as features of significant sites. We investigated five indices of fractionation to assess the consistency of signal classification, and associated the effects of ablation lesions to these classification techniques.

Methods 16 patients undergoing first-time CA of persAF were studied. A NavX CFAE map was acquired after circumferential pulmonary vein isolation. EGM data were exported and analysed in custom software. CA was performed on sites showing high ContA. Offline analysis compared 5 algorithms: ContA, OI, Dominant Frequency (DF), CFAEmean (NavX equivalent) and shortest complex interval (SCI, Carto equivalent) after signal screening. The effect of ablation lesions on AF cycle length was correlated with underlying fractionation.

Results CA on ContA sites terminated AF in 50\% cases. 2589 8-s EGMs and 471 ablation lesions targeting fractionation were analysed. Varying the refractory periods (RP) of algorithms produced large changes in signal classification for CFAEmean (Pearson $\mathrm{R}=0.81 \pm 0.05$ for $10 \mathrm{~ms}$ variation in $\mathrm{RP}, \mathrm{R}=0.08$ for $90 \mathrm{~ms}$ variation in $\mathrm{RP})$ and SCI $(R=0.95 \pm 0.04$ for $10 \mathrm{~ms}, \mathrm{R}=-0.34$ for $90 \mathrm{~ms})$, whereas Cont $A$ was unaffected $(R=0.99 \pm 0.01$ for $10 \mathrm{~ms}, R=0.80$ for 